International Journal of Environmental Research and

Public Health

ISSN 1660-4601

www.mdpi.com/journal/ijerph

Article

\title{
In-situ Real-Time Monitoring of Volatile Organic Compound Exposure and Heart Rate Variability for Patients with Multiple Chemical Sensitivity
}

\section{Atsushi Mizukoshi ${ }^{1,2, *}$, Kazukiyo Kumagai ${ }^{3}$, Naomichi Yamamoto ${ }^{4}$, Miyuki Noguchi ${ }^{5}$, Kazuhiro Yoshiuchi ${ }^{6}$, Hiroaki Kumano ${ }^{2,7}$, Kou Sakabe ${ }^{8}$ and Yukio Yanagisawa ${ }^{9}$}

1 Department of Environmental Medicine and Behavioral Science, Kinki University Faculty of Medicine, 377-2, Ohno-higashi, Osakasayama, Osaka 589-8511, Japan

2 Institute of Applied Brain Sciences, Waseda University, 2-579-15 Mikajima, Tokorozawa, Saitama 359-1192, Japan; E-Mail: hikumano@waseda.jp

3 Environmental Health Laboratory, California Department of Public Health, 850 Marina Bay Pkwy, Richmond, CA 94804, USA; E-Mail: kkumagai@cdph.ca.gov

4 Department of Environmental Health Sciences, Graduate School of Public Health, Seoul National University, 1 Gwanak-ro, Gwanak-gu, Seoul 151-742, Korea; E-Mail: nyamamoto@snu.ac.kr

5 Department of Materials and Life Science, Faculty of Science and Technology, Seikei University, 3-3-1 Kichijoji-kitamachi, Musashino, Tokyo 180-8633, Japan; E-Mail: noguchi@ejs.seikei.ac.jp

6 Department of Stress Sciences and Psychosomatic Medicine, Graduate School of Medicine, The University of Tokyo, Hongo 7-3-1, Bunkyo-ku, Tokyo 113-8655, Japan;

E-Mail: kyoshiuc-tky@umin.ac.jp

7 Faculty of Human Sciences, Waseda University, 2-579-15 Mikajima, Tokorozawa, Saitama 359-1192, Japan

8 Department of Anatomy and Cellular Biology, Tokai University School of Medicine, 143 Shimokasuya, Isehara, Kanagawa 259-1193, Japan; E-Mail: sakabek@tokai-u.jp

9 The University of Tokyo, 7-3-1, Hongo, Bunkyo-ku, Tokyo 113-8654, Japan; E-Mail: yukio@kaiseigakuen.jp

* Author to whom correspondence should be addressed; E-Mail: mizukoshi@med.kindai.ac.jp; Tel.: +81-72-366-0221 (ext. 3275); Fax: +81-72-368-1192.

Academic Editor: Paul B. Tchounwou

Received: 25 August 2015 / Accepted: 28 September 2015 / Published: 5 October 2015 


\begin{abstract}
In-situ real-time monitoring of volatile organic compound (VOC) exposure and heart rate variability (HRV) were conducted for eight multiple chemical sensitivity (MCS) patients using a VOC monitor, a Holter monitor, and a time-activity questionnaire for $24 \mathrm{~h}$ to identify the relationship between VOC exposure, biological effects, and subjective symptoms in actual life. The results revealed no significantly different parameters for averaged values such as VOC concentration, HF (high frequency), and LF (low frequency) to HF ratio compared with previous data from healthy subjects (Int. J. Environ. Res. Public Health 2010, 7, 4127-4138). Significant negative correlations for four subjects were observed between HF and amounts of VOC change. These results suggest that some patients show inhibition of parasympathetic activities along with VOC exposure as observed in healthy subjects. Comparing the parameters during subjective symptoms and normal condition, VOC concentration and/or VOC change were high except for one subject. HF values were low for five subjects during subjective symptoms. Examining the time-series data for VOC exposure and HF of each subject showed that the subjective symptoms, VOC exposure, and HF seemed well related in some symptoms. Based on these characteristics, prevention measures of symptoms for each subject may be proposed.
\end{abstract}

Keywords: real-time monitoring; MCS; VOC; HRV

\title{
1. Introduction
}

Multiple chemical sensitivity (MCS) has been defined as an acquired disorder characterized by recurrent symptoms, referable to multiple organ systems, occurring in response to demonstrable exposure to many chemically unrelated compounds at doses far below those established in the general population to cause harmful effects [1]. Medical researchers and clinicians from the United States and Canada signed the 1999 Consensus on MCS and established the following criteria: (1) The symptoms are reproducible with (repeated chemical) exposure; (2) The condition is chronic; (3) Low levels of exposure (lower than previously or commonly tolerated) result in manifestations of the syndrome; (4) The symptoms improve or resolve when the incitants are removed; (5) Responses occur to multiple chemically unrelated substances; (6) Symptoms involve multiple organ systems [2].

As defined above, chemical exposure has been assumed to trigger the symptoms, although the underlying mechanism of MCS remains disputed. Therefore, it is inevitable to investigate the relationship between chemical exposure and symptoms for understanding the pathogenesis, making a diagnosis, and proposing a measure of cure and prevention of symptoms. Provocation challenges have been conducted to clarify whether the patients actually show different responses to low levels of exposure [3]. These tests are conducted in controlled environments. However, in actual life where various chemicals exist, it is assumed that "masking" (acclimatization, apposition, and addiction) may hide the exposure-symptom relationships [4]. As a result, exposure-symptom relationships in actual lives may be different from those in controlled environments. It is therefore essential to understand the actual conditions of MCS measurements of chemical exposure and symptoms in actual life. Few studies have been done measuring chemical exposure of patients in actual lives and evaluating the relationship to the 
symptoms. Shinohara et al. [5] measured the exposures of $15 \mathrm{MCS}$ patients to both carbonyl compounds and volatile organic compounds (VOCs) that may induce hypersensitive reaction in actual lives. The results demonstrated that the chemicals responsible for hypersensitive reactions varied from patient to patient. Moreover, the concentration during symptoms was far below the WHO indoor guidelines. Saito et al. [6] used Ecological Momentary Assessment to monitor everyday symptoms in addition to the environmental chemical exposure measurement. The results showed that some causative chemicals were detected in 11 of 14 MCS patients and 11 physical symptoms and four mood subscales were significantly aggravated when they experienced hypersensitivity symptoms. In those studies, chemical compounds were collected by adsorbents and the composition and concentration were analyzed using gas chromatography-mass spectrometry (GCMS) and high-performance liquid chromatography (HPLC). These integration analysis tests are general methods to measure the concentration of volatile organic compound (VOC) components and the result obtained by this method is the average concentration during a certain period (e.g., from $30 \mathrm{~min}$ to 1 week).

To elucidate the responsible exposure for each symptom, it is necessary to detect the fluctuation of personal exposure because personal exposure fluctuates according to personal activities and locations in a short period. However, it is difficult to obtain the personal exposure fluctuation from the data of average concentration. On the other hand, measurement methods with higher time resolution such as real-time monitoring using portable VOC monitors to measure total VOC concentrations [7-9] provide time-series data of personal exposure concentrations, but do not elucidate the VOC components. Therefore, it is assumed that using these monitors the fluctuation of personal exposure can be detected and the information about the context or simultaneity between exposure and symptoms of MCS patients can be obtained.

To investigate the relationship between the fluctuation of VOC exposure and its biological effects, it is necessary to know the change in biological parameters in a relatively short time. Since MCS patients usually report various autonomic nerve symptoms, it is desirable to know the temporal changes in autonomic nerve function in actual life. Heart rate variability (HRV) measured by Holter monitor has been used to evaluate the biological effects caused by environmental factors [10-17].

In our previous study, VOC exposure concentrations and HRV using VOC and Holter monitors were measured for seven healthy subjects [18]. In this study, we applied this method to MCS patients and identified characteristics of the relationship between VOC exposure, biological effects, and subjective symptoms in actual lives. In this paper, first, VOC exposure and HRV parameters of MCS patients were compared to controls. Moreover, the correlations between VOC exposure and HRV parameters were considered. Further, the parameters during subjective symptom and normal condition were compared. Finally, time-series data for each subject were observed in detail.

\section{Experimental Section}

\subsection{Study Design}

This study was designed to simultaneously monitor personal VOC concentrations and HRV for eight MCS patients under usual daily life conditions. The measurements were conducted from 2006 to 2007. The subjects were requested to wear the Holter monitor, carry the VOC monitor, and record the time-activity logs during monitoring. 
All subjects gave their informed consent for inclusion before they participated in the study. The study was conducted in accordance with the Declaration of Helsinki, and the protocol was approved by the Research Ethical Committee of the Kitasato Institute Hospital of No.13 D-180-10.

\subsection{Subjects}

The subjects were eight MCS patients including three adult males and five adult females, and the ages ranged from 31 to 62 years ( $44 \pm 11$ years). These patients consulted doctors in the Division of Environmental Medical Center, Kitasato Institute Hospital. Various examinations for diagnosis, including neuro-ophthalmologic examination, medical examination by interview, and questionnaire survey were performed in a clean room. The questionnaire included the reason for visiting, subjective symptoms, questions about life environment, and the Quick Environment Exposure Sensitivity Inventory (QEESI) in Japanese [19]. The patients were diagnosed with MCS by medical specialists from the comprehensive results of these examinations.

Because the responsible chemical compounds are expected to be different for each patient, it is difficult to lump MCS patients together. Therefore, MCS patients were limited to those advanced from Sick Building Syndrome whose responsible compounds were considered to be VOCs.

\subsection{VOC Monitoring}

VOC exposure concentrations were measured for $24 \mathrm{~h}$ by a portable real-time VOC monitor with photo ionization detector (PID) (ppbRAE plus; RAE Systems, San Jose, CA, USA). Detailed information on VOC monitoring has been described in a previous study [18]. Before each measurement, calibrations were conducted using 10 or $100 \mathrm{ppm}$ isobutylene gas. Temperature and relative humidity (RH) were measured by a thermo-hygrometer (HOBO; Onset Computer Corporation, Jackson, MS, USA) carried with the VOC monitor.

For the analysis, VOC concentration averaged over each 5-min interval and the changes in VOC concentration amount in 5-min interval $(\triangle \mathrm{VOC})$, calculated by subtracting the minimum value from the maximum value of each interval, were used. Additionally, the differential changes of 5-min averaged VOC concentration from the previous 5-min averaged VOC concentration were calculated and divided into positive values $(\mathrm{d}+$ VOC $)$ and negative values $(\mathrm{d}-\mathrm{VOC})$. Temperature and RH, averaged over each 5-min interval, were also used for the analysis.

\subsection{HRV Analysis}

The continuous electrocardiogram (ECG) data were recorded for $24 \mathrm{~h}$ by the Holter monitor (FM-150 or FM-180; Fukuda Denshi, Tokyo, Japan). Detailed information on HRV analysis has been described in a previous study [18]. To avoid eliciting a response to the electrode seals, they were exposed to air before use and dispelled their smell as much as possible.

For the analysis, high frequency (HF) and low frequency (LF) power were averaged over 5-min intervals, HF power was used as an indicator of parasympathetic activity, and the power ratio of 5-min averaged LF to 5-min averaged HF (LF/HF) was used as an indicator of sympathetic activity [20,21]. 


\subsection{Time-Activity Pattern}

The time-activity patterns were recorded by the subjects (Figure 1). The subjects were requested to select their locations and activities in each 5-min interval from the following alternatives: four kinds of locations including home, office, other indoor, and outdoor; and six kinds of personal activities including sitting, standing, walking, exercising, eating, and sleeping. In addition, when a symptom was induced, subjects were instructed to indicate the symptom level on a $0-10$ scale $(0=$ not at all a problem, $5=$ moderate symptoms, and $10=$ disabling symptoms) and select the type from the symptom severity items in QEESI [22,23] including musculoskeletal, airway/mucous membranes, heart/chest-related, gastrointestinal, cognitive, affective, neuromuscular, head-related, skin, and genitourinary. Responsible exposure chemicals or events could be written in the remarks column.

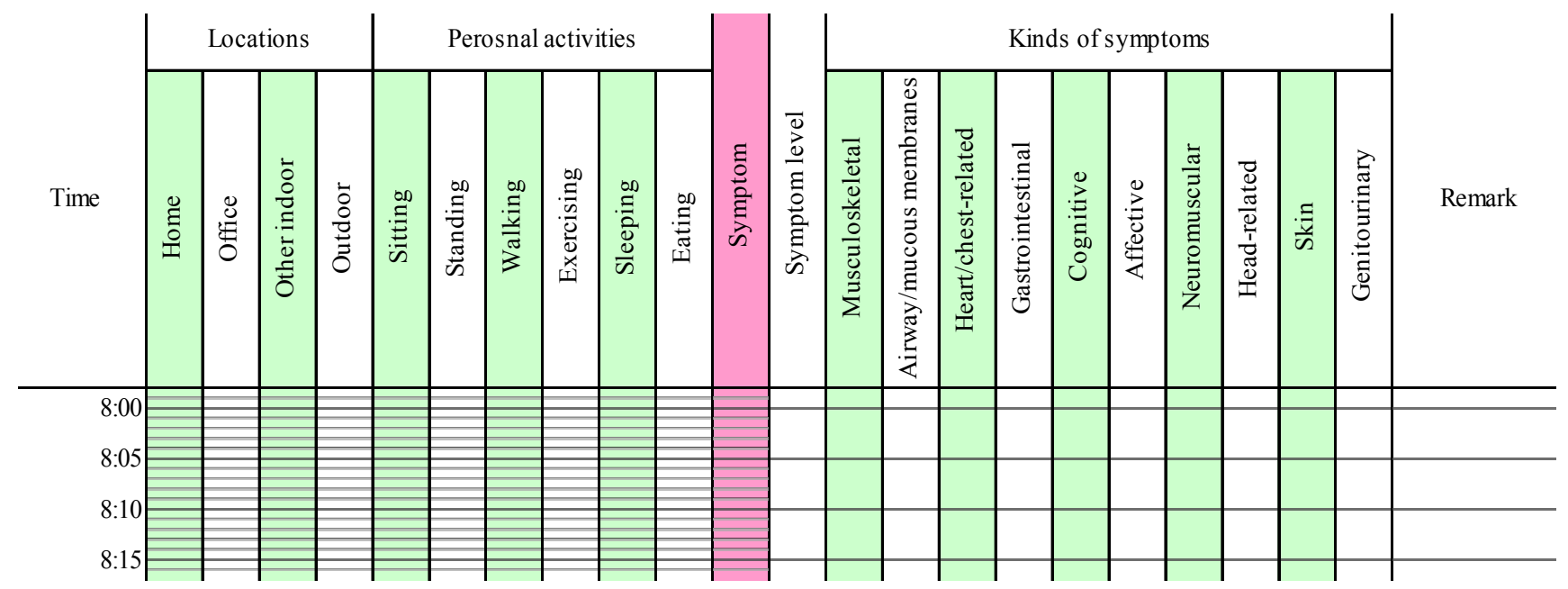

Figure 1. An example of a time-activity log sheet.

To ignore the confounding factors, we excluded the data of the time spent for these activities (i.e., exercising, eating, and sleeping) and the duration of the effect (i.e., $15 \mathrm{~min}$ after exercising and $1 \mathrm{~h}$ after eating) from the analyses in the same manner as the previous study [18].

\subsection{Statistical Analysis}

VOC exposure concentration and HRV parameters of MCS patients were compared to controls using Wilcoxon non-parametric test. To assess the relationships between VOC exposures and HRV parameters Spearman rank correlation coefficients were calculated. Time ratios of respective symptoms were analyzed using principal component analysis to figure out the characteristics of subjective symptoms. The parameters during subjective symptom and normal condition were compared using Wilcoxon non-parametric test. All analysis were conducted using IBM SPSS Statistics Version 22 (IBM).

\section{Results}

\subsection{Statistical Summary}

Table 1 shows a summary of VOC exposure concentrations and HRV parameters observed for all subjects who participated in this study. The results indicate that exposure concentrations differed for 
each patient. Table 1 also shows control (healthy subject). Only the data from a previous study [18] was used. No significant difference was observed between patients and healthy subject for all the parameters (Wilcoxon non-parametric test).

Table 1. Summary of VOC exposure concentrations and HRV parameters for patients and controls.

\begin{tabular}{ccccccc}
\hline \multirow{2}{*}{ Parameters } & \multicolumn{3}{c}{ Patients } & & Controls $^{\mathbf{c}}$ & \multirow{2}{*}{$\boldsymbol{p}^{\mathbf{d}}$} \\
\cline { 2 - 4 } & $\mathbf{n}^{\mathbf{a}}$ & Mean $\pm \mathbf{S D}^{\mathbf{b}}$ & & $\mathbf{n}$ & Mean $\pm \mathbf{S D}$ & \\
\hline Total & 8 & $306 \pm 148$ & 7 & $176 \pm 130$ & 0.12 \\
Home & 8 & $262 \pm 204$ & 7 & $299 \pm 267$ & 1.00 \\
\hline $\log _{10} \mathrm{HF}\left(\mathrm{m} \cdot \mathrm{sec}^{2}\right)$ & 8 & $1.5 \pm 0.2$ & 7 & $1.7 \pm 0.4$ & 0.34 \\
$\mathrm{LF} / \mathrm{HF}$ & 8 & $3.8 \pm 1.9$ & 7 & $3.8 \pm 2.2^{\mathrm{e}}$ & 1.00 \\
\hline
\end{tabular}

${ }^{\mathbf{a}}$ Sample size; ${ }^{\mathbf{b}}$ Standard deviation; ${ }^{\mathbf{c}}$ Data from a previous study $[18] ;{ }^{\mathbf{d}}$ Wilcoxon non-parametric test; ${ }^{\mathrm{e}} \mathrm{LF} / \mathrm{HF}$ was calculated using LF and HF averaged over 5-min intervals.

\subsection{Bivariate Analysis}

Spearman rank correlation coefficients were calculated to assess the relationships between VOC exposures and HRV parameters measured within the same 5-min intervals. Table 2 shows a summary of the correlations for all subjects. The sex and age of these subjects are also listed in Table 2 .

Table 2. Correlations between VOC exposure and HRV parameters.

\begin{tabular}{|c|c|c|c|c|c|c|c|c|c|c|}
\hline \multirow{2}{*}{ Parameters } & \multicolumn{8}{|c|}{ Subject } & \multirow{4}{*}{$-d$} & \multirow{4}{*}{$+^{e}$} \\
\hline & $\mathbf{A}$ & B & $\mathbf{C}$ & D & $\mathbf{E}$ & $\mathbf{F}$ & $\mathbf{G}$ & $\mathbf{H}$ & & \\
\hline Sex (M: male, F: female) & $\mathbf{M}$ & $\mathbf{F}$ & $\mathbf{F}$ & $\mathbf{F}$ & $\mathbf{M}$ & $\mathbf{M}$ & $\mathbf{F}$ & $\mathbf{F}$ & & \\
\hline Age (years) & 39 & 62 & 33 & 46 & 31 & 49 & 35 & 54 & & \\
\hline VOC vs. HF & $-0.35 * *$ a & $0.19 *$ & -0.03 & -0.00 & $-0.34 * *$ & -0.04 & 0.06 & -0.13 & $6(2)$ & $2(1)$ \\
\hline$\Delta \mathrm{VOC} v s . \mathrm{HF}$ & $-0.38 * *$ & 0.01 & 0.11 & $-0.32 * *$ & 0.07 & -0.03 & $-0.24 *$ & $-0.30 * *$ & $5(4)$ & $3(0)$ \\
\hline $\mathrm{d}+\mathrm{VOC} v s . \mathrm{HF}$ & $-0.46 * *$ & -0.17 & 0.13 & $-0.37 *$ & 0.02 & 0.04 & $-0.38 *$ & $-0.45 * *$ & $5(4)$ & $3(0)$ \\
\hline $\mathrm{d}-\mathrm{VOC} v s . \mathrm{HF}$ & $0.31 * b$ & -0.08 & 0.09 & $0.45 * *$ & $0.30 * *$ & $0.39 * *$ & 0.15 & $0.34 * *$ & $1(0)$ & $7(5)$ \\
\hline VOC vs. LF/HF & 0.06 & $-0.22 *$ & 0.01 & -0.16 & $0.26 * *$ & -0.04 & -0.06 & 0.01 & $4(1)$ & $4(1)$ \\
\hline$\Delta \mathrm{VOC} v s . \mathrm{LF} / \mathrm{HF}$ & 0.08 & 0.11 & 0.02 & $0.28 * *$ & -0.09 & -0.08 & $0.29 * *$ & 0.04 & $2(0)$ & $6(2)$ \\
\hline $\mathrm{d}+\mathrm{VOC} v s . \mathrm{LF} / \mathrm{HF}$ & 0.04 & 0.10 & 0.27 & $0.45 * *$ & 0.04 & -0.18 & 0.27 & 0.12 & $1(0)$ & $7(1)$ \\
\hline $\mathrm{d}-\mathrm{VOC} v s . \mathrm{LF} / \mathrm{HF}$ & 0.13 & -0.21 & -0.13 & $-0.33 *$ & $-0.27 * *$ & $-0.30 *$ & -0.21 & -0.08 & $7(3)$ & $1(0)$ \\
\hline Temp vs. HF & -0.01 & $0.38 * *$ & -0.07 & 0.13 & $-{ }^{c}$ & 0.15 & $0.21 *$ & $-0.40 * *$ & $3(1)$ & $4(2)$ \\
\hline RH vs. HF & 0.10 & $-0.61 * *$ & 0.15 & 0.18 & - & $-0.21 *$ & 0.16 & $0.38 * *$ & $2(2)$ & $5(1)$ \\
\hline Temp vs. LF/HF & $0.20 *$ & -0.12 & -0.02 & -0.03 & - & -0.04 & -0.12 & 0.09 & $5(0)$ & $2(1)$ \\
\hline $\mathrm{RH} v s . \mathrm{LF} / \mathrm{HF}$ & -0.08 & -0.15 & -0.14 & $-0.22 *$ & - & 0.18 & $-0.20 *$ & -0.10 & $6(2)$ & $1(0)$ \\
\hline
\end{tabular}

$\mathrm{a} * *$ Spearman rank correlation, $p<0.01 ; \mathrm{b} *$ Spearman rank correlation, $p<0.05 ;{ }^{\mathrm{c}}$ Data not obtained;

d - Numbers of the subjects showing negative correlation (significant); ${ }^{\mathbf{e}}+$ Numbers of the subjects showing positive correlation (significant). 


\subsection{Characteristics of Subjective Symptoms}

Table 3 shows subjective symptom times and time ratios of respective symptoms for all subjects. These counted all symptom times in a 5-min interval including those during exercising, eating, and sleeping. Average time of symptom was $3.2 \pm 3.0(0.3-9.8) \mathrm{h}$. The order of frequency of symptoms is airway/mucous membranes ( $8 / 8$ subjects), heart/chest-related (6/8 subjects), gastrointestinal (5/8 subjects), cognitive ( $4 / 8$ subjects), neuromuscular ( $4 / 8$ subjects), head-related ( $4 / 8$ subjects), musculoskeletal (3/8 subjects), affective ( $3 / 8$ subjects), skin ( $2 / 8$ subjects), and genitourinary ( $1 / 8$ subjects). Principal component analysis was performed on the variance-covariance matrix ( 8 subjects $\times 10$ variables), where the variables comprised the time ratios of respective symptoms. Figure 2 shows principal component 1 (PC1) and principal component 2 (PC2) scores for each subject, which accounted for $78 \%$ variance. This result indicates the characteristics of symptoms of subject $\mathrm{A}, \mathrm{B}$, and $\mathrm{H}$ were different from other subjects.

Table 3. Subjective symptom time (h) and time ratio of respective symptoms (\%).

\begin{tabular}{ccccccccc}
\hline \multirow{2}{*}{ Symptoms } & \multicolumn{10}{c}{ Subject } \\
\cline { 2 - 9 } & A & B & C & D & E & F & G & H \\
\hline Symptom time & 2.7 & 9.8 & 4.8 & 0.8 & 2.8 & 0.3 & 2.4 & 2.1 \\
Musculoskeletal & 0 & 8 & 0 & 0 & 52 & 0 & 0 & 4 \\
Airway/mucous membranes & 94 & 84 & 7 & 22 & 3 & 75 & 97 & 84 \\
Heart/chest-related & 0 & 84 & 24 & 44 & 0 & 25 & 17 & 92 \\
Gastrointestinal & 0 & 5 & 40 & 22 & 3 & 0 & 0 & 4 \\
Cognitive & 0 & 81 & 5 & 0 & 0 & 0 & 31 & 84 \\
Affective & 0 & 1 & 2 & 0 & 0 & 0 & 0 & 4 \\
Neuromuscular & 91 & 92 & 10 & 0 & 0 & 0 & 0 & 12 \\
Head-related & 94 & 92 & 43 & 0 & 18 & 0 & 0 & 0 \\
Skin & 0 & 2 & 0 & 0 & 0 & 0 & 0 & 64 \\
Genitourinary & 0 & 0 & 0 & 0 & 0 & 0 & 7 & 0 \\
\hline
\end{tabular}

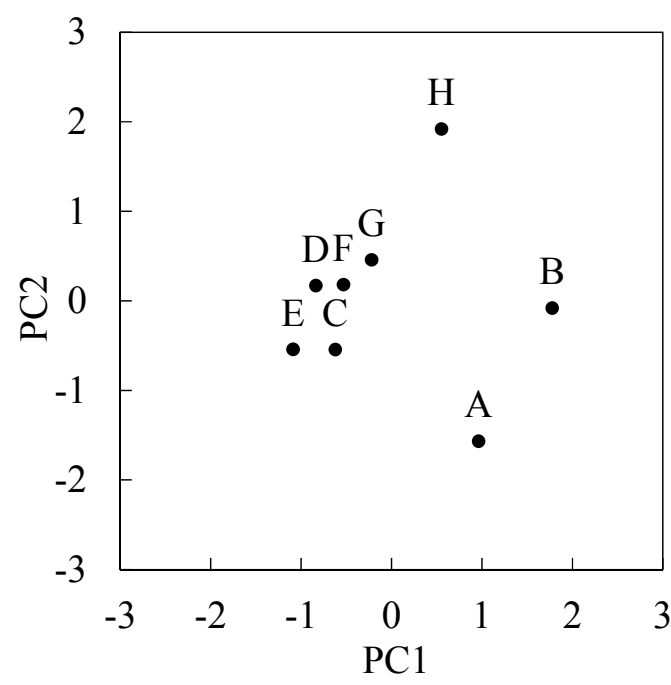

Figure 2. Plot of first principal component (PC1) and second principal component (PC2) scores of eight subjects. 


\subsection{Comparison between Subjective Symptom Time and Normal Time}

Table 4 compares subjective symptom times and normal condition times. Because the symptom of subject F was observed for only one interval (5-min) after excluding confounding factors, the data is eliminated.

Table 4. Comparison of the parameter of subjective symptom time and normal condition time.

\begin{tabular}{|c|c|c|c|c|c|c|c|c|c|}
\hline \multirow{2}{*}{ Parameters } & \multicolumn{7}{|c|}{ Subject } & \multirow{2}{*}{$\downarrow^{f}$} & \multirow{2}{*}{$\uparrow^{\mathrm{g}}$} \\
\hline & $\mathbf{A}$ & B & $\mathrm{C}$ & D & $\mathbf{E}$ & G & H & & \\
\hline VOC & $\downarrow^{\mathrm{a}}$ & $\uparrow * *$ & $\downarrow$ & $\uparrow$ & $\uparrow *$ & $\uparrow$ & $\uparrow$ & $2(0)$ & $5(2)$ \\
\hline$\Delta \mathrm{VOC}$ & $\uparrow^{b}$ & $\uparrow$ & $\uparrow$ & $\downarrow$ & $\uparrow *$ & $\uparrow$ & $\downarrow$ & $2(0)$ & $5(1)$ \\
\hline HF & $\downarrow * * \mathrm{c}$ & $\downarrow * *$ & $\downarrow * *$ & $\downarrow$ & $\uparrow * *$ & $\uparrow$ & $\downarrow$ & $5(3)$ & $2(1)$ \\
\hline $\mathrm{LF} / \mathrm{HF}$ & $\uparrow * \mathrm{~d}$ & $\downarrow$ & $\uparrow * *$ & $\uparrow$ & $\downarrow$ & $\downarrow$ & $\downarrow$ & $4(0)$ & $3(2)$ \\
\hline Temp & $\downarrow$ & $\downarrow$ & $\uparrow *$ & $\downarrow$ & - e & $\downarrow * *$ & $\downarrow * *$ & $5(2)$ & $1(1)$ \\
\hline RH & $\uparrow * *$ & $\uparrow * *$ & $\uparrow$ & $\uparrow$ & - & $\uparrow *$ & $\uparrow *$ & $0(0)$ & $6(4)$ \\
\hline
\end{tabular}

${ }^{\mathrm{a}} \downarrow$ Average value was lower during symptoms; ${ }^{\mathrm{b}} \uparrow$ Average value was higher during symptoms; ${ }^{\mathrm{c}} * *$ Wilcoxon non-parametric test, $p<0.01 ;{ }^{\mathrm{d} *}$ Wilcoxon non-parametric test, $p<0.05$; ${ }^{\mathrm{e}}$ Data not obtained; ${ }^{\mathrm{f}}$ Number of the subjects showing lower during symptoms (significant); ${ }^{\mathrm{g}}$ Number of the subjects showing higher during symptoms (significant).

\section{Discussion}

\subsection{Comparisons of VOC Exposure and HRV Parameters between Patients and Controls}

It is assumed that patients avoid exposure to chemicals, and consequently their exposure level of chemical compounds is lower than that of healthy subjects [5]. However, the patients' VOC exposure concentration in total was higher than that of controls in this study although the differences were not significant (Table 1). The VOC concentration that were measured by the VOC monitor used in this study included various VOCs. Thus, chemicals that did not induce symptoms in patients may have been included in the measurements, which could explain why the VOC exposure concentrations of patients were not lower than those in healthy subjects. In addition, it should be taken into consideration that the subjects were recruited from volunteers and were not representative of a random sample of controls or MCS patients. With regard to HRV parameters, HF of patients had a low tendency although the differences were not significant.

\subsection{Correlations between VOC Exposure and HRV Parameters}

Significant negative correlation in two out of eight subjects and significant positive correlation in one out of eight subjects were observed between VOC concentration and HF (Table 2), suggesting there is no consistent trend between VOC concentration and HF for MCS patients. On the other hand, significant negative correlations between $\triangle \mathrm{VOC}$ and HF were observed in four out of eight subjects, suggesting that changes of VOC concentrations in 5-min intervals were associated with decreased activity of parasympathetic nervous system. In addition, significant negative correlations between $\mathrm{d}+\mathrm{VOC}$ and HF were observed in four out of eight subjects, and significant positive correlations between $\mathrm{d}-\mathrm{VOC}$ and HF were seen in five out of eight subjects. These results indicate that HF tends to be low when the VOC concentrations increase or decrease. If VOC exposure is the cause of decreased HF, it is assumed 
that decreased VOC exposure enhances HF power. However, HF was low when the VOC concentration decreased. Based on the existence of a causal relationship between exposure and the parasympathetic nervous system, one possibility is to assume that decrease of VOC exposure as with increase causes the $\mathrm{HF}$ decrease. Another possibility is that a delay of HF increases after VOC exposure. Between LF/HF and $\triangle \mathrm{VOC}$, significant positive correlation was observed in two out of eight subjects. Significant positive correlations were observed between $d+\mathrm{VOC}$ and LF/HF in one out of eight subjects, and significant negative correlations were observed between $d-$ VOC and LF/HF in three out of eight subjects. These tendencies were opposite to HF.

As in the previous study, negative correlations between $\triangle \mathrm{VOC}$ and HF in six out of seven healthy subjects were observed. Since these tendencies were more frequent in healthy subjects than in patients, there is a possibility that the absence of significant correlation between $\triangle \mathrm{VOC}$ and HRV parameters is characteristic of MCS patients.

\subsection{Comparison between Subjective Symptom Time and Normal Time}

During subjective symptoms compared to normal conditions, VOC concentration was higher in five subjects and $\triangle \mathrm{VOC}$ was higher in five subjects [significant differences were observed in subject $\mathrm{B}$ and E (Wilcoxon non-parametric test)] (Table 4). That is, VOC concentration and/or the change amount were high in all subjects except for subject F. In relation to HRV, the values of HF in five subjects were low during subjective symptoms (significant differences were observed in three subjects). These tendencies suggested the presence of high VOC concentration or change and low HF power when the subjects feel symptoms. In addition, the RH was high during subjective symptoms in six subjects, suggesting that RH has some relationship to subjective symptoms.

\subsection{Case Studies}

The measurement in this study was not designed to clarify the causal linkage between exposure and symptoms, but the context or simultaneity between exposure and symptoms can be observed from the time-series data. Therefore, time-series data for each subject were observed in detail in each case. To grasp the tendencies visually, average and maximum VOC concentration during 1 min and $\log _{10} \mathrm{HF}$ during $1 \mathrm{~min}$ and 15-min moving average of $\log _{10} \mathrm{HF}$ are indicated. Here it was assumed that the symptoms occurred when the subject sensed the exposure of chemicals. The longtime delay of symptom occurrence after exposure was not considered. This is based on the survey which clarified that the timing of symptom occurrence was almost immediately after an exposure [24].

Moreover, from this information, preventive measures were proposed for each subject. There is no common MCS treatment protocol accepted across medical disciplines. Gibson et al. surveyed perceived treatment efficacy for conventional and alternative therapies reported by a person with MCS. As a result, participants rated chemical avoidance, creating a chemical-free living space, and prayer as the three most useful interventions [25]. On the other hand, cognitive therapy, such as mindfulness, are being explored as treatment option for MCS [26,27].

This study includes several limitations attributed to various confounding factors based on the measurements in actual lives. VOC monitors measure the concentration of a wide variety of environmental VOCs in total, including non-symptom-related VOCs. Moreover, HRV parameters are 
affected by various environmental factors and personal activities. In addition, the relationship between exposure and symptoms cannot refer to causal relationship. However, this method provided numerically-expressed data for actual condition of MCS exposure and symptoms which had been assumed depending on the interview and suggested a new insight into treatment processes.

\subsubsection{Subject A}

Subject A was a 39-year-old male and a researcher. Responsible exposures during monitoring were insecticides, tobacco, cosmetics, refresher, paint, detergent, smoke, and disinfectants inside a building. Figure 3 shows time-series data of VOC concentration and $\log _{10} \mathrm{HF}$ of subject A. In the time from 18:30 to $0: 30$, several peak shape VOC concentration changes were observed and almost simultaneously (just before or immediately after the peak exposure) the subject felt the symptoms. This indicates that symptoms were induced when he was exposed to some VOCs (although symptoms before acute exposure may be anticipatory symptoms). It is noteworthy that $\log _{10} \mathrm{HF}$ decreased before the occurrence of symptoms during exposure to relatively high and successive concentrations indoors (e.g., 21:00-21:30). From these findings, the exposure and symptom relationship is assumed as below: when HF is decreasing, a symptom is induced along with sudden elevation of VOC concentration. Meanwhile, a symptom occurred without high concentration of VOC, for example, during 16:20-17:10.

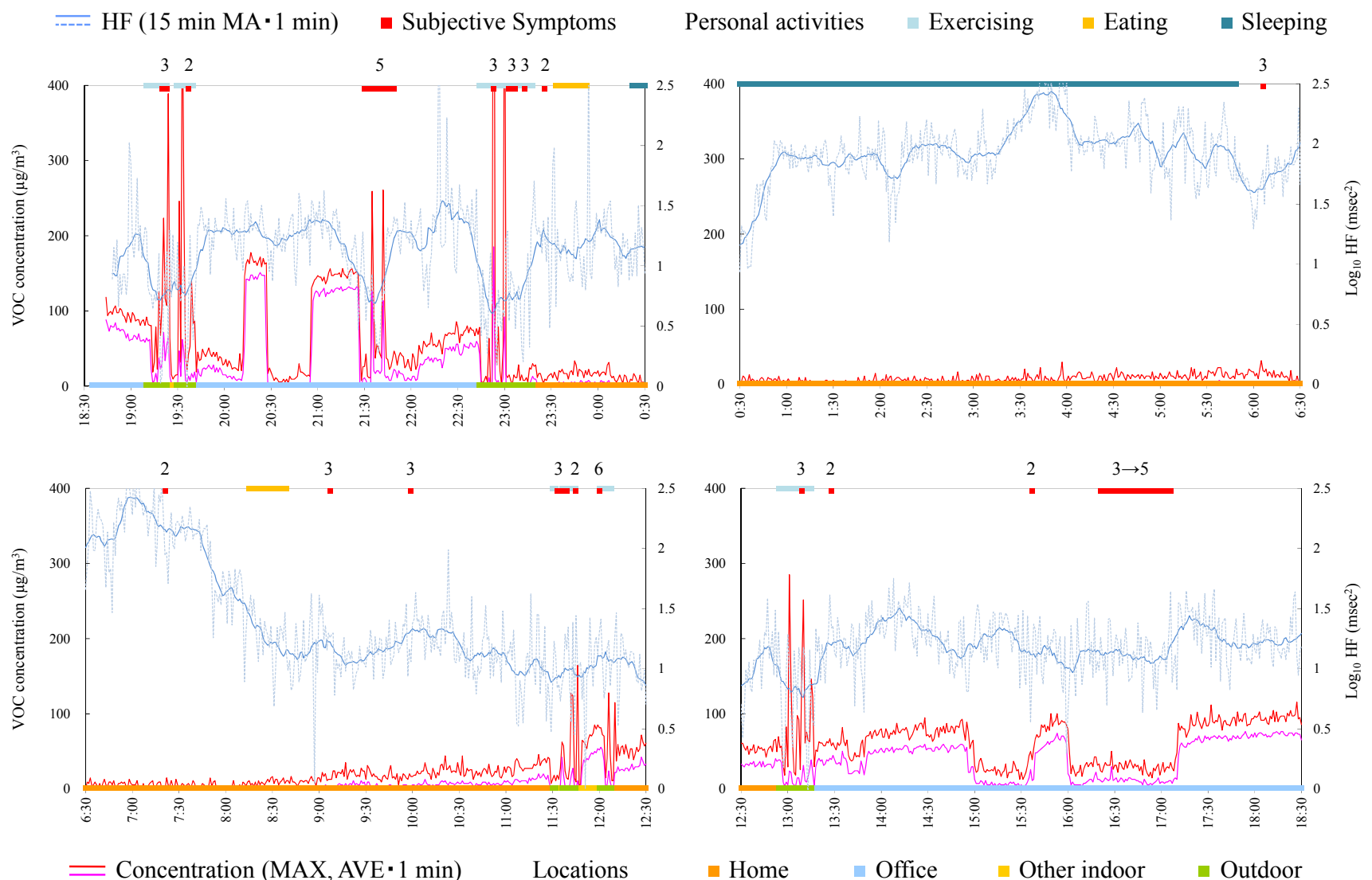

Figure 3. Time-series data of VOC concentration and $\log _{10}$ HF (subject A). Subjective symptoms and personal activities are indicated in upper side and locations are indicated in lower side. The values on subjective symptoms are symptom levels. 
Consequently, since the symptom of this subject seemed synchronized with acute exposure, the measure to prevent symptoms for this subject is to avoid the condition or environment where the acute exposure of chemical or the environment (particularly outdoors) occurs. In addition, enhancing the parasympathetic nerve activities in daily life may be effective.

\subsubsection{Subject B}

Subject B was a 62-year-old female and a homemaker. Figure 4 shows time-series data of VOC concentration and $\log _{10}$ HF of subject B. During 8:00-8:30, a peak exposure was observed and at the same time symptoms were induced. However, the symptoms occurred frequently during 8:30-13:00, although the concentration of VOC hardly fluctuated. Therefore, in this case it was suggested that the subject mostly felt symptoms without increased VOC concentration. This suggests that the VOC monitor could not measure the concentration or concentration change of the chemicals that induced symptoms in this subject. For example the VOC monitor used in this study cannot detect formaldehyde which was rated as causing most symptomatology in persons self-identified with MCS [28]. Alternatively, this suggests that the subject repeated learned symptoms that could have been induced by a similar environment or condition in which symptoms were previously provoked by specific chemicals. HF during symptoms was significantly lower than that during normal conditions (Table 4). From this standpoint, other measures except for avoiding exposure, such as neurological treatment or cognitive therapy, may be effective.
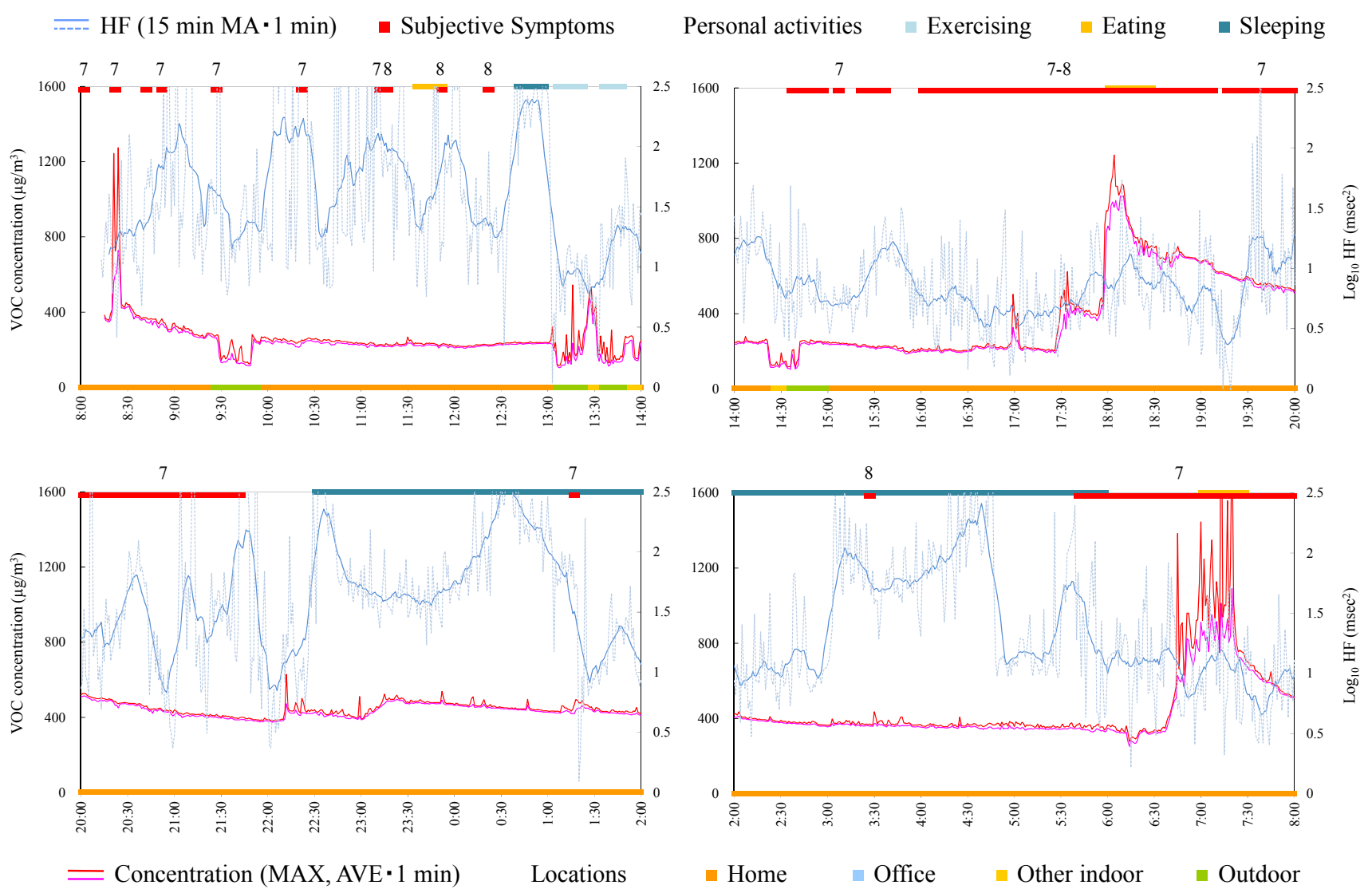

Figure 4. Time-series data of VOC concentration and $\log _{10}$ HF (subject B). Subjective symptoms and personal activities are indicated in upper side and locations are indicated in lower side. The values on subjective symptoms are symptom levels. 


\subsubsection{Subject C}

Subject C was a 33-year-old female and a homemaker. Since symptoms worsened during measurement, the Holter monitor was removed at 19:30. Responsible exposures during monitoring were car exhaust, open burning, pesticide, tobacco, personal computer, and stove. Figure 5 shows time-series data of VOC concentration and $\log _{10} \mathrm{HF}$ of subject $\mathrm{C}$. The concentration in the house was relatively higher than that in other environments because the subject lived in new residential housing. In fact, measurement of VOCs in breath in the clean room indicated a high concentration of $\alpha$-pinene, suggesting that $\alpha$-pinene emitted from new wood material was absorbed by the body. Since the symptoms were not always induced in the house, it was suggested that VOCs existing on a steady basis in the house, including $\alpha$-pinene, were not possible compounds for this subject. At 14:45 a symptom was induced in a car by open burning and almost simultaneously increase in VOC concentration was detected. Severe symptom was induced at 18:40 when a guest came. The concentration of VOC rapidly decreased and increased. This may be because of incursion of outdoor air by opening the front door. Because VOC concentration of outdoor air was low compared with indoor air, the concentration increase by responsible compounds could not be detected. This is a limitation of the measurement using the VOC monitor, which cannot separate the components.

HF during symptoms was significantly lower than during normal condition (Table 4) and some symptoms seem to be induced after HF decreases. Therefore, taking care to live a life enhancing the parasympathetic nerve activity may be effective also for this subject.

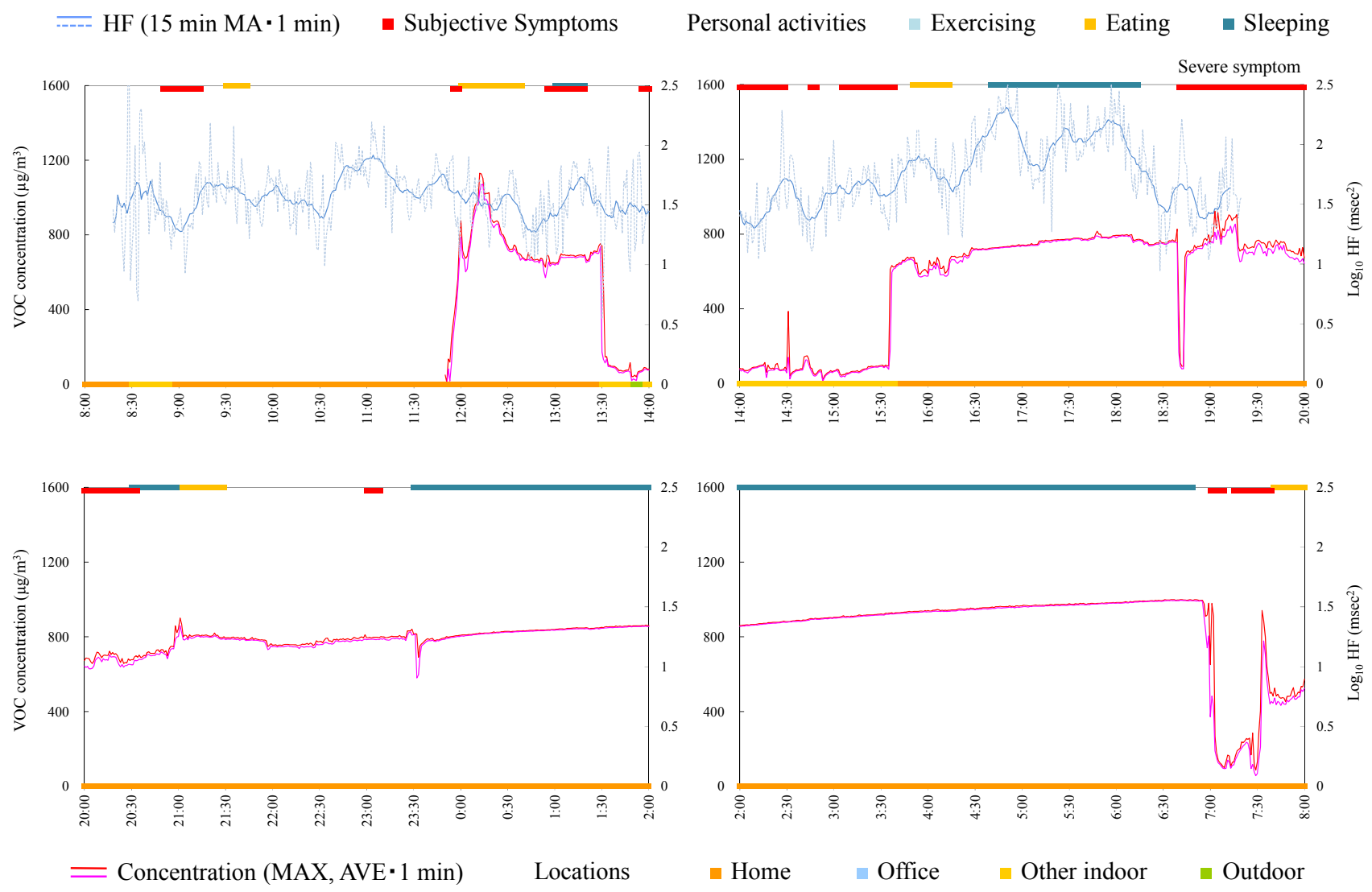

Figure 5. Time-series data of VOC concentration and $\log _{10}$ HF (subject C). Subjective symptoms and personal activities are indicated in upper side and locations are indicated in lower side. The values on subjective symptoms are symptom levels. 


\subsubsection{Subject D}

Subject D was a 46-year-old female and a homemaker. Figure 6 shows time-series data of VOC concentration and $\log _{10}$ HF of subject D. After 20:00, the subject developed symptoms of flu. As is the case with subject A, sets of tendencies (after HF decrease, the subjective symptom, and acute increase of VOC concentration) were observed during 13:30-14:00 and 15:30-16:30. Therefore, avoiding the condition or environment of acute exposure and enhancing the parasympathetic nerve activities may be effective for prevention of symptoms.
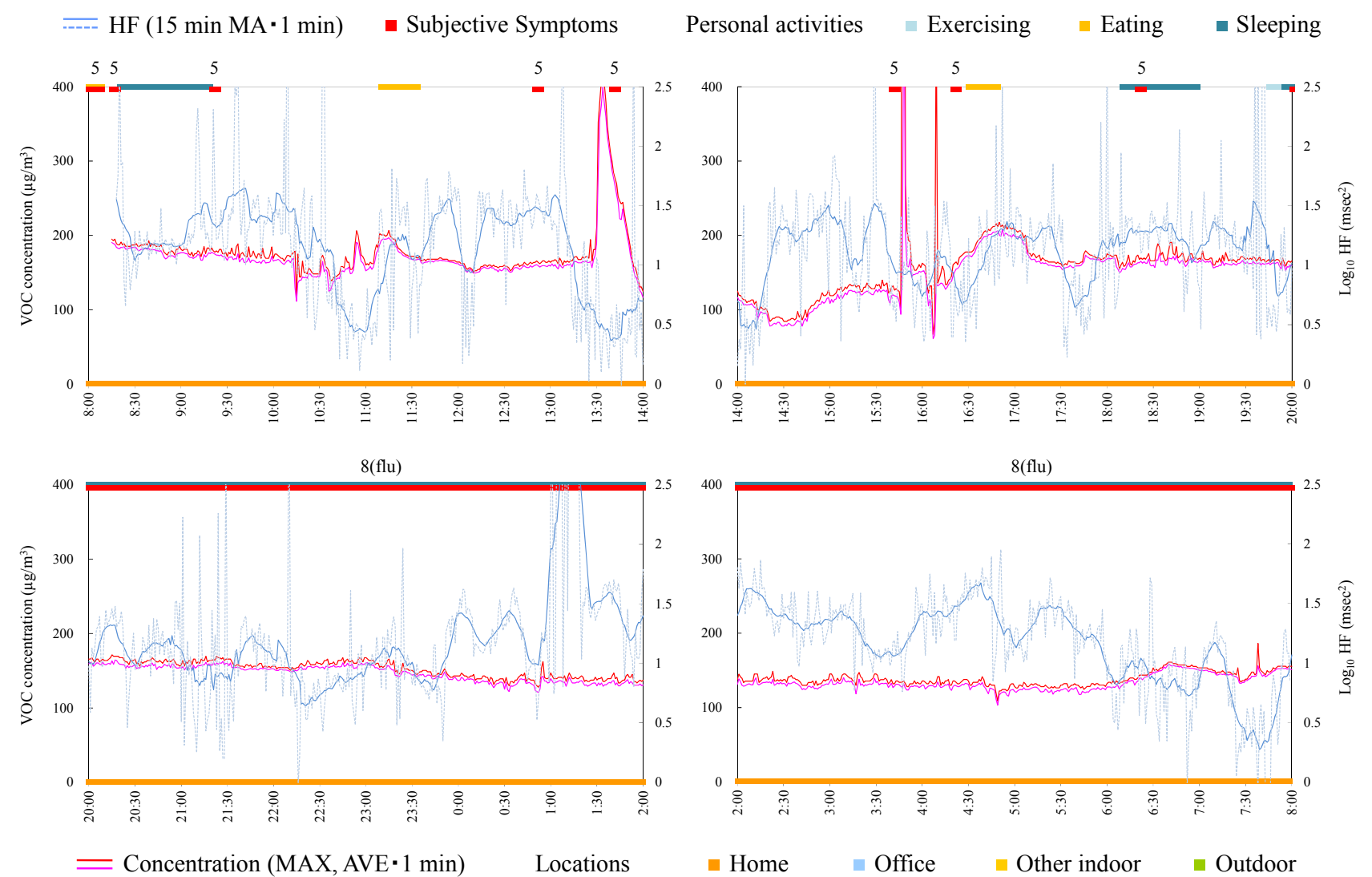

Figure 6. Time-series data of VOC concentration and $\log _{10} \mathrm{HF}$ (subject D). Subjective symptoms and personal activities are indicated in upper side and locations are indicated in lower side. The values on subjective symptoms are symptom levels.

\subsubsection{Subject E}

Subject E was a 31-year-old male and an office worker. Figure 7 shows time-series data of VOC concentration and $\log _{10}$ HF of subject E. HF was significantly high during symptoms (Table 4). The symptom from 11:00 was induced while the VOC concentration was increased in office. Therefore, creating a chemical-free living space may be necessary. 


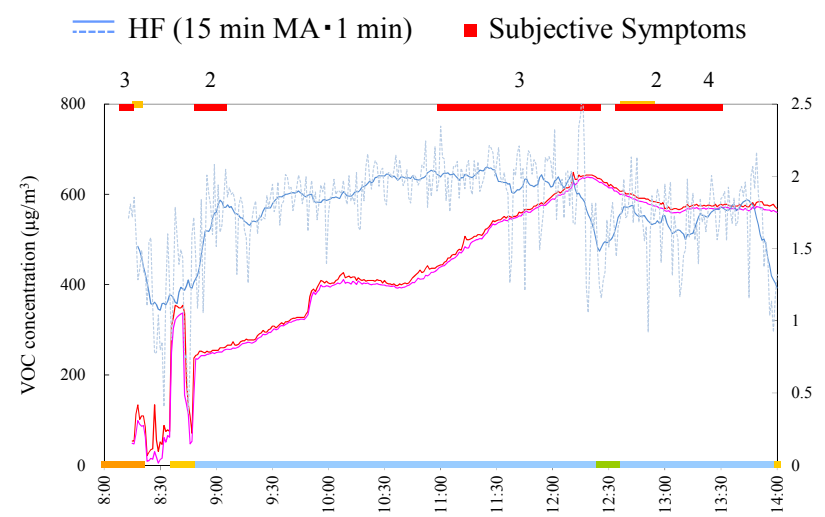

Personal activities $\square$ Exercising $\square$ Eating $\square$ Sleeping
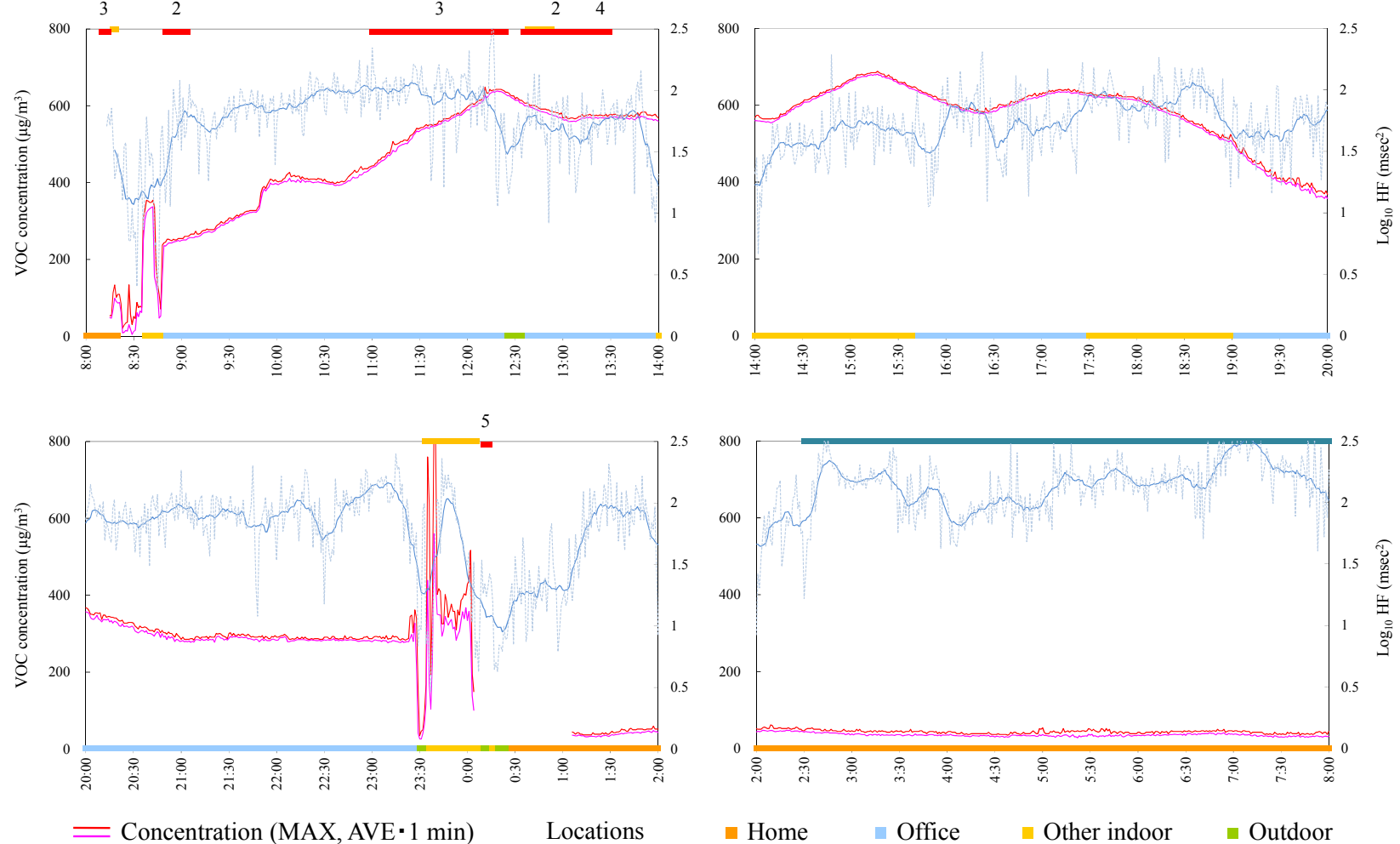

Figure 7. Time-series data of VOC concentration and $\log _{10}$ HF (subject E). Subjective symptoms and personal activities are indicated in upper side and locations are indicated in lower side. The values on subjective symptoms are symptom levels.

\subsubsection{Subject $F$}

Subject $\mathrm{F}$ was a 49-year-old male and an office worker. Responsible exposures during monitoring were liquid detergents used for cleaning and tobacco. Figure 8 shows time-series data of VOC concentrations and $\log _{10} \mathrm{HF}$ of subject $\mathrm{F}$. The symptoms were induced by relatively low concentrations at 9:40 and 13:40. After HF was decreased by exercise, the subjective symptom appeared during slight change of VOC concentration at 20:45. Meanwhile, symptoms were not induced by VOC concentrations by large increase and fluctuation from 15:30 and from 18:00. Therefore, avoiding the responsible exposures, such as detergents and tobacco, may be effective to prevent the symptoms for this subject. 

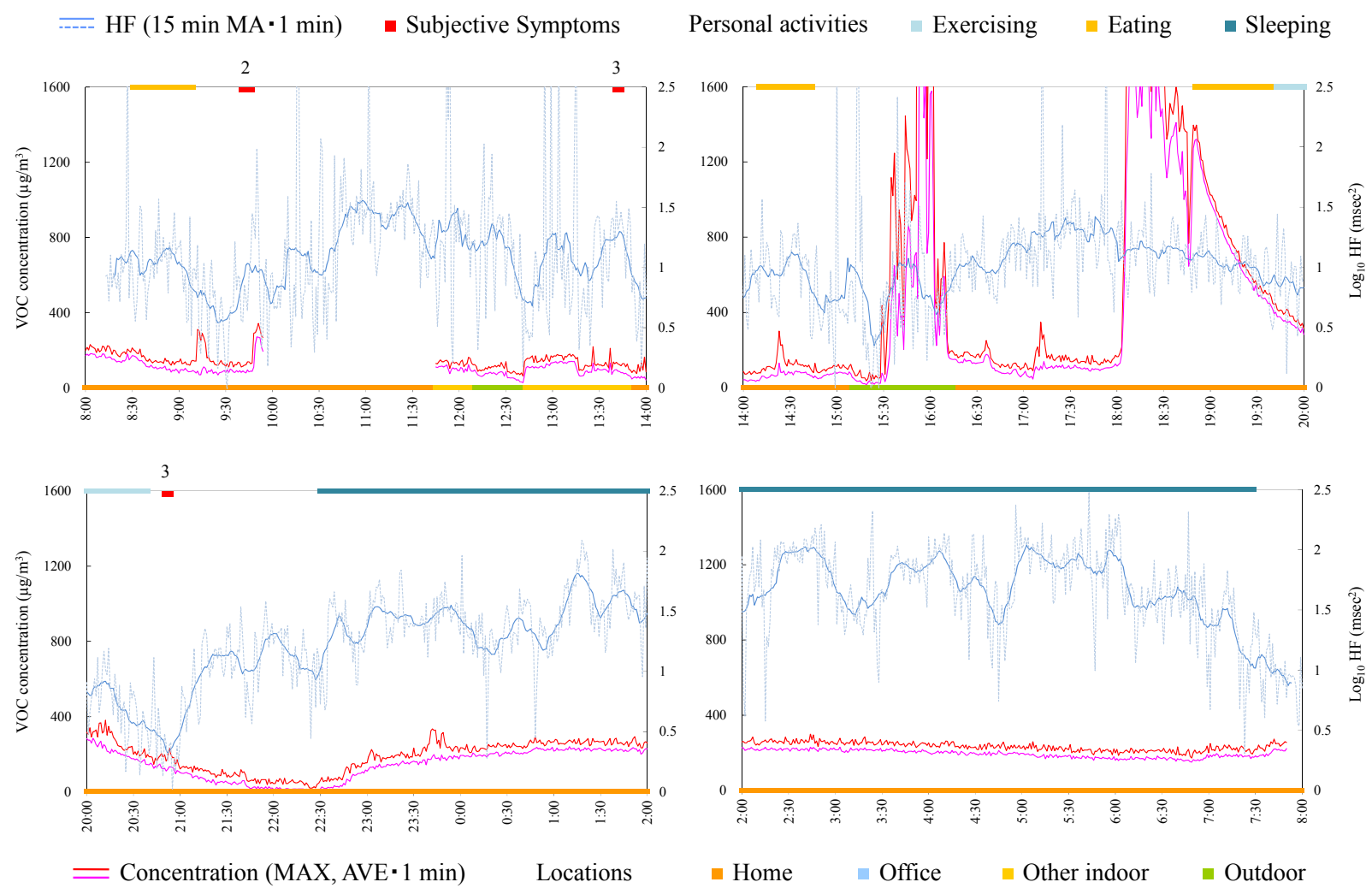

Figure 8. Time-series data of VOC concentration and $\log _{10} \mathrm{HF}$ (subject F). Subjective symptoms and personal activities are indicated in upper side and locations are indicated in lower side. The values on subjective symptoms are symptom levels.

\subsubsection{Subject $\mathrm{G}$}

Subject $G$ was a 35-year-old female and on leave from her job. Responsible exposures during monitoring were odor of new organic cotton, bag for measurement apparatus, print, paper, pencil, ballpoint pen, envelope, odor of detergents, odor of drugstore, odors of cosmetics, perfume, and tobacco, odors of clothes and hair dressing, exhaust gas, smoke, fragrance, and odors of the dryer and closet. Figure 9 shows time-series data of VOC concentration and $\log _{10} \mathrm{HF}$ of subject G. VOC concentration was very high during daytime, in the home, after breakfast (8:50). Consistent tendencies were not observed between VOC exposure, symptoms, and HF. Because the symptoms in this subject were mainly induced by odors of consumer products, which may affect only a local space and may not reach to the monitor sufficiently, there was a possibility that the monitor could not detect their effects. 

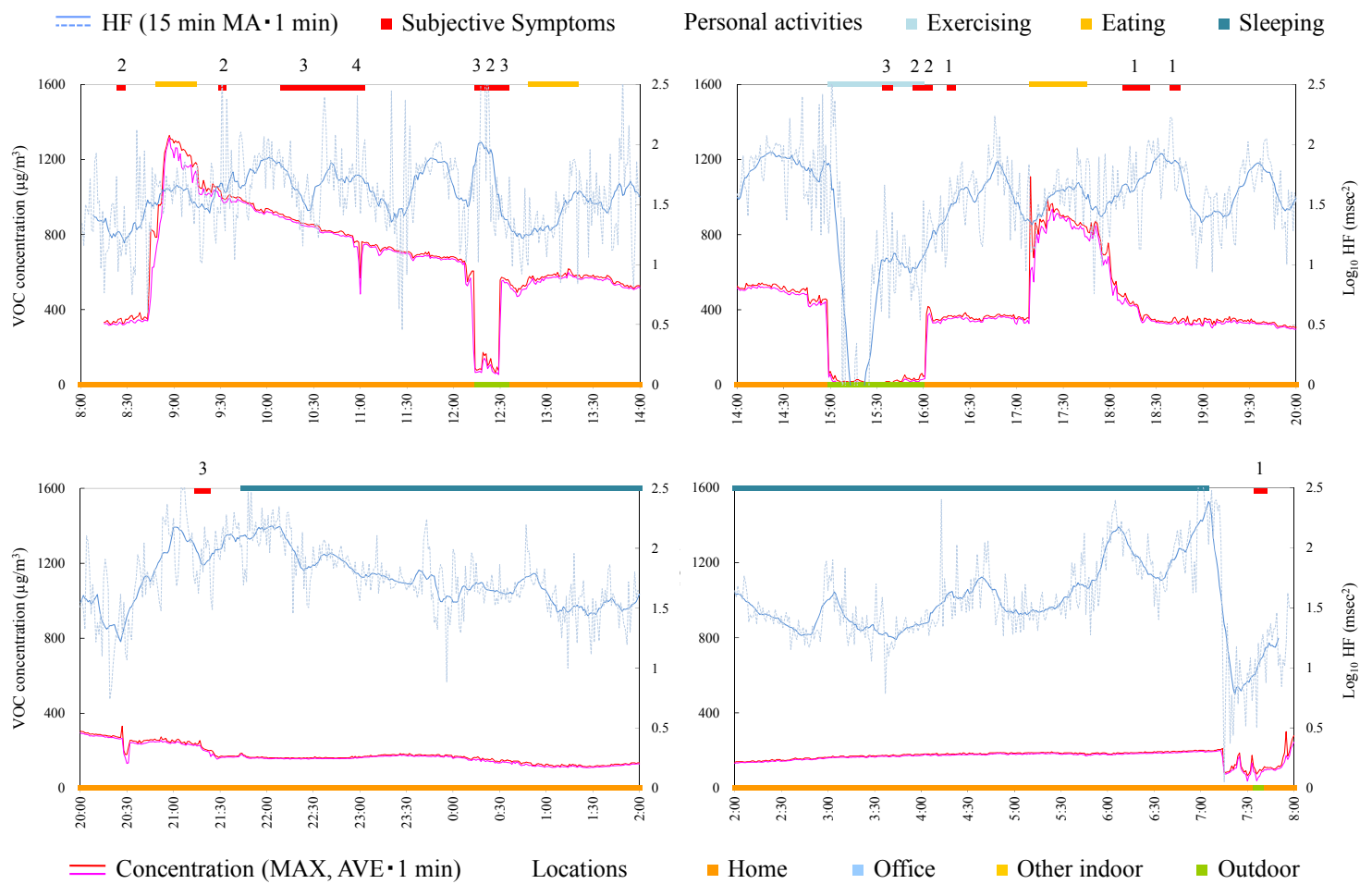

Figure 9. Time-series data of VOC concentration and $\log _{10}$ HF (subject G). Subjective symptoms and personal activities are indicated in upper side and locations are indicated in lower side. The values on subjective symptoms are symptom levels.
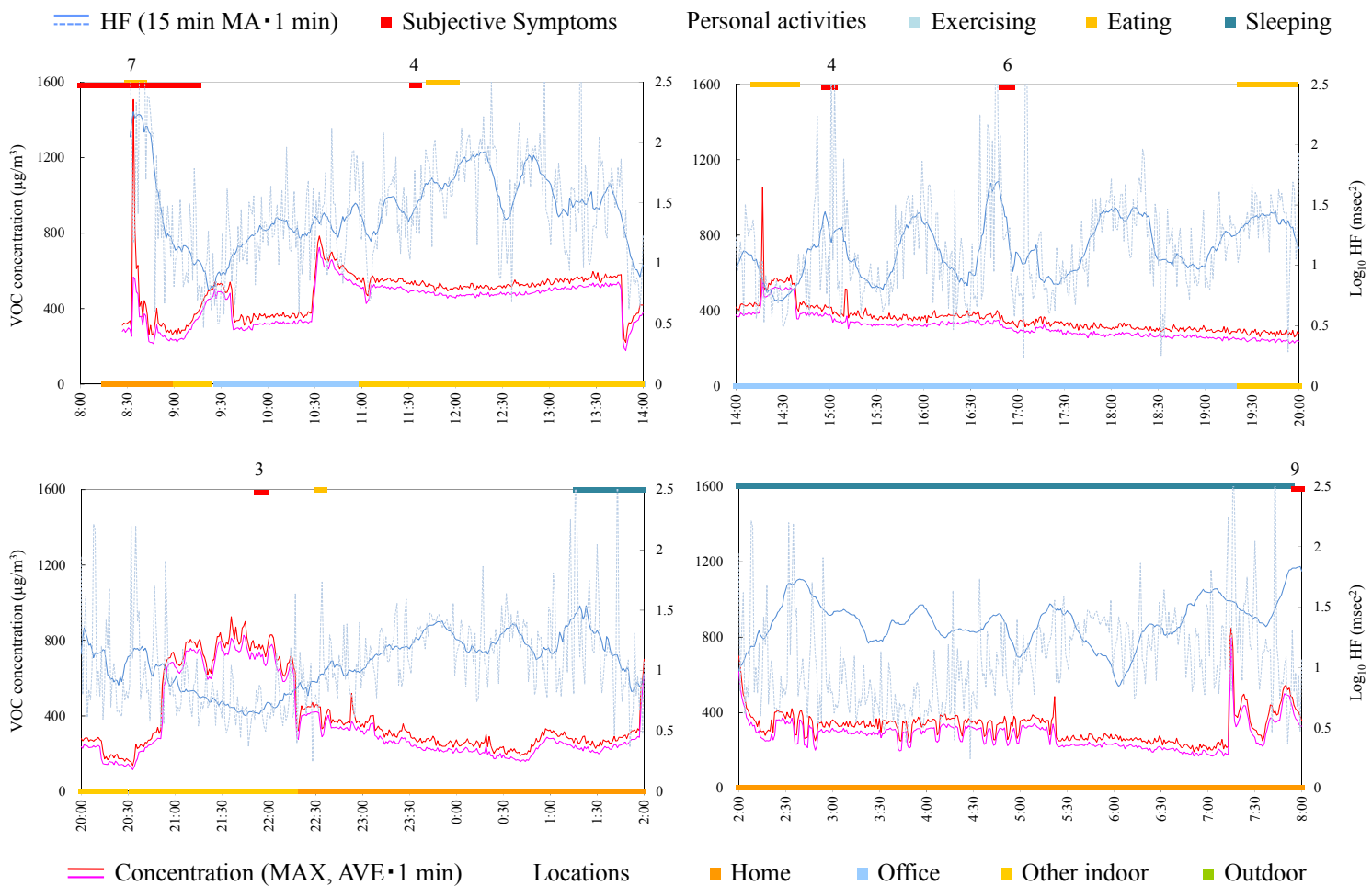

Figure 10. Time-series data of VOC concentration and $\log _{10} \mathrm{HF}$ (subject H). Subjective symptoms and personal activities are indicated in upper side and locations are indicated in lower side. The values on subjective symptoms are symptom levels. 


\subsubsection{Subject $\mathrm{H}$}

Subject $\mathrm{H}$ was a 54-year-old female and a company owner. Figure 10 shows time-series data of VOC concentration and $\log _{10} \mathrm{HF}$ of subject $\mathrm{H}$. After decrease in HF, the subjective symptom appeared during relatively high concentration at 21:55. For other symptoms, consistent tendencies were not observed between VOC exposure, symptoms, and HF.

\section{Conclusions}

In-situ real-time monitoring of VOC exposure and HRV were conducted for eight MCS patients using a VOC monitor, a Holter monitor, and a time-activity pattern for $24 \mathrm{~h}$ to identify the relationship between VOC exposure, biological effects, and subjective symptoms of MCS patients in actual life. The results showed that there were no significantly different parameters for averaged values such as VOC exposure concentration, HF, and LF/HF compared with previous data from healthy subjects. Between HF and VOC change amount, significant negative correlations for four out of eight subjects were observed. These results suggest that some patients show inhibition of parasympathetic activities along with VOC exposure as seen with healthy subjects in a previous study. Comparing the parameters during subjective symptoms and normal conditions, VOC concentrations and/or VOC change amounts were high in all subjects except for one, and the values of HF were low for five subjects during subjective symptoms, suggesting the presence of high VOC concentration or change and low HF power when the subjects feel symptoms. Examining the time-series data for VOC exposure and $\log _{10}$ HF of each subject revealed subjects whose subjective symptoms, VOC exposure, and HF seemed well related and other subjects whose findings did not appear related. Though there are limitations of the study design and the method, characteristics of relationship between exposure and symptoms were suggestive, and based on these characteristics, prevention measures of symptoms for each subject may be proposed.

\section{Acknowledgments}

We thank Takako Matsui (Akita University Graduate School of Medicine) and Manabu Ozawa (Kitasato University Kitasato Institute Hospital). We are grateful to all the subjects who participated in this study. This study was supported by Health Labour Sciences Research Grant and Grant-in-Aid for Scientific Research (A) 19201007. Part of this research was conducted by Tokyo Metropolitan Collaboration of Regional Entities for the Advancement of Technological Excellence, Japan Science and Technology Agency (JST). This study was also supported by a health science research grant from the Japan Ministry of the Environment.

\section{Author Contributions}

Kazukiyo Kumagai, Naomichi Yamamoto, and Miyuki Noguchi were responsible for the VOC measurements and analysis. Kazuhiro Yoshiuchi and Hiroaki Kumano were responsible for HRV measurement and analysis. Kou Sakabe was responsible for subject selection and recruitment. Yukio Yanagisawa had the original idea for the study. Atsushi Mizukoshi wrote this paper. All the authors contributed to the draft of the manuscript, read, and approved the final manuscript. 


\section{Conflicts of Interest}

The authors declare no conflict of interest. The founding sponsors had no role in the design of the study; in the collection, analyses, or interpretation of data; in the writing of the manuscript; and in the decision to publish the results.

\section{References}

1. Cullen, M.R. The worker with multiple chemical sensitivities: An overview. Occup. Med. 1987, 2, 655-661.

2. McCready, P. Multiple chemical sensitivity: A 1999 consensus. Arch. Environ. Health 1999, 54, 147-149.

3. Das-Munshi, J.; Rubin, G.J.; Wessely, S. Multiple chemical sensitivities: A systematic review of provocation studies. J. Allergy Clin. Immunol. 2006, 118, 1257-1264.

4. Miller, C.S. Chemical sensitivity: Symptom, syndrome or mechanism for disease? Toxicology 1996, $111,69-86$.

5. Shinohara, N.; Mizukoshi, A.; Yanagisawa, Y. Identification of responsible volatile chemicals that induce hypersensitive reactions to multiple chemical sensitivity patients. J. Expo. Anal. Environ. Epidemiol. 2004, 14, 84-91.

6. Saito, M.; Kumano, H.; Yoshiuchi, K.; Kokubo, N.; Ohashi, K.; Yamamoto, Y.; Shinohara, N.; Yanagisawa, Y.; Sakabe, K.; Miyata, M.; et al. Symptom profile of multiple chemical sensitivity in actual life. Psychosom. Med. 2005, 67, 318-325.

7. Coy, J.D.; Bigelow, P.L.; Buchan, R.M.; Tessari, J.D.; Parnell, J.O. Field evaluation of a portable photoionization detector for assessing exposure to solvent mixtures. AIHAJ 2000, 61, 268-274.

8. Oka, K.; Iizuka, A.; Inoue, Y.; Mizukoshi, A.; Noguchi, M.; Yamasaki, A.; Yanagisawa, Y. Development of a combined real time monitoring and integration analysis system for volatile organic compounds (VOCs). Int. J. Environ. Res. Public Health 2010, 7, 4100-4110.

9. Hori, H.; Ishimatsu, S.; Fueta, Y.; Ishidao, T. Evaluation of a real-time method for monitoring volatile organic compounds in indoor air in a Japanese university. Environ. Health Prev. Med. 2013, 18, 285-292.

10. Liao, D.; Creason, J.; Shy, C.; Williams, R.; Watts, R.; Zweidinger, R. Daily variation of particulate air pollution and poor cardiac autonomic control in the elderly. Environ. Health Perspect. 1999, $107,521-525$.

11. Chan, C.C.; Chuang, K.J.; Shiao, G.M.; Lin, L.Y. Personal exposure to submicrometer particles and heart rate variability in human subjects. Environ. Health Perspect. 2004, 112, 1063-1067.

12. Chuang, K.J.; Chan, C.C.; Chen, N.T.; Su, T.C.; Lin, L.Y. Effects of particle size fractions on reducing heart rate variability in cardiac and hypertensive patients. Environ. Health Perspect. 2005, 113, 1693-1697.

13. Pope, C.A., 3rd; Eatough, D.J.; Gold, D.R.; Pang, Y.; Nielsen, K.R.; Nath, P.; Verrier, R.L.; Kanner, R.E. Acute exposure to environmental tobacco smoke and heart rate variability. Environ. Health Perspect. 2001, 109, 711-716. 
14. Riojas-Rodriguez, H.; Escamilla-Cejudo, J.A.; Gonzalez-Hermosillo, J.A.; Tellez-Rojo, M.M.; Vallejo, M.; Santos-Burgoa, C.; Rojas-Bracho, L. Personal PM2.5 and CO exposures and heart rate variability in subjects with known ischemic heart disease in Mexico City. J. Expo. Sci. Environ. Epidemiol. 2006, 16, 131-137.

15. Sandstrom, M.; Lyskov, E.; Hornsten, R.; Hansson Mild, K.; Wiklund, U.; Rask, P.; Klucharev, V.; Stenberg, B.; Bjerle, P. Holter ECG monitoring in patients with perceived electrical hypersensitivity. Int. J. Psychophysiol. 2003, 49, 227-235.

16. Weichenthal, S.; Kulka, R.; Belisle, P.; Joseph, L.; Dubeau, A.; Martin, C.; Wang, D.; Dales, R. Personal exposure to specific volatile organic compounds and acute changes in lung function and heart rate variability among urban cyclists. Environ. Res. 2012, 118, 118-123.

17. Mehta, A.J.; Adam, M.; Schaffner, E.; Barthelemy, J.C.; Carballo, D.; Gaspoz, J.M.; Rochat, T.; Schindler, C.; Schwartz, J.; Zock, J.P.; et al. Heart rate variability in association with frequent use of household sprays and scented products in SAPALDIA. Environ. Health Perspect. 2012, 120, 958-964.

18. Mizukoshi, A.; Kumagai, K.; Yamamoto, N.; Noguchi, M.; Yoshiuchi, K.; Kumano, H.; Yanagisawa, Y. A novel methodology to evaluate health impacts caused by VOC exposures using real-time VOC and Holter monitors. Int. J. Environ. Res. Public Health 2010, 7, 4127-4138.

19. Ishikawa S.; Miyata, M. Multiple Chemical Sensitivity: Criteria and test methods for diagnosis. Allergol. Immuol. 1999, 6, 990-998.

20. Heart Rate Variability. Standards of Measurement, Physiological Interpretation, and Clinical Use. Task Force of the European Society of Cardiology and the North American Society of Pacing and Electrophysiology. Available online: http://www.escardio.org/static_file/Escardio/Guidelines/ Scientific-Statements/guidelines-Heart-Rate-Variability-FT-1996.pdf (accessed on 25 August 2015).

21. Malliani, A.; Lombardi, F.; Pagani, M. Power spectrum analysis of heart rate variability: A tool to explore neural regulatory mechanisms. Br. Heart J. 1994, 71, 1-2.

22. Miller, C.S.; Prihoda, T.J. The Environmental Exposure and Sensitivity Inventory (EESI): A standardized approach for measuring chemical intolerances for research and clinical applications. Toxicol. Ind. Health 1999, 15, 370-385.

23. Miller, C.S.; Prihoda, T.J. A controlled comparison of symptoms and chemical intolerances reported by Gulf War veterans, implant recipients and persons with multiple chemical sensitivity. Toxicol. Ind. Health 1999, 15, 386-397.

24. Caress, S.M.; Steinemann, A.C. A review of a two-phase population study of multiple chemical sensitivities. Environ. Health Perspect. 2003, 111, 1490-1497.

25. Gibson, P.R.; Elms, A.N.; Ruding, L.A. Perceived treatment efficacy for conventional and alternative therapies reported by persons with multiple chemical sensitivity. Environ. Health Perspect. 2003, 111, 1498-1504.

26. Sampalli, T.; Berlasso, E.; Fox, R.; Petter, M. A controlled study of the effect of a mindfulness-based stress reduction technique in women with multiple chemical sensitivity, chronic fatigue syndrome, and fibromyalgia. J. Multidiscip. Healthc. 2009, 2, 53-59.

27. Hauge, C.R.; Bonde, P.J.; Rasmussen, A.; Skovbjerg, S. Mindfulness-based cognitive therapy for multiple chemical sensitivity: A study protocol for a randomized controlled trial. Trials 2012, 13, doi:10.1186/1745-6215-13-179. 
28. Gibson, P.R.; Vogel, V.M. Sickness-related dysfunction in persons with self-reported multiple chemical sensitivity at four levels of severity. J. Clin. Nurs. 2009, 18, 72-81.

(C) 2015 by the authors; licensee MDPI, Basel, Switzerland. This article is an open access article distributed under the terms and conditions of the Creative Commons Attribution license (http://creativecommons.org/licenses/by/4.0/). 